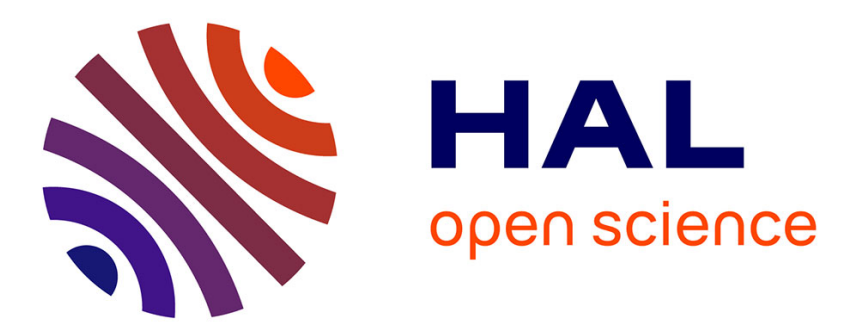

\title{
Dielectric function and optical properties of Al-rich AlInN alloys pseudomorphically grown on GaN
}

\author{
E Sakalauskas, H Behmenburg, C Hums, P Schley, G Rossbach, C Giesen, M
} Heuken, H Kalisch, R H Jansen, J Bläsing, et al.

\section{- To cite this version:}

E Sakalauskas, H Behmenburg, C Hums, P Schley, G Rossbach, et al.. Dielectric function and optical properties of Al-rich AlInN alloys pseudomorphically grown on GaN. Journal of Physics D: Applied Physics, 2010, 43 (36), pp.365102. 10.1088/0022-3727/43/36/365102 . hal-00569694

\section{HAL Id: hal-00569694 https://hal.science/hal-00569694}

Submitted on 25 Feb 2011

HAL is a multi-disciplinary open access archive for the deposit and dissemination of scientific research documents, whether they are published or not. The documents may come from teaching and research institutions in France or abroad, or from public or private research centers.
L'archive ouverte pluridisciplinaire HAL, est destinée au dépôt et à la diffusion de documents scientifiques de niveau recherche, publiés ou non, émanant des établissements d'enseignement et de recherche français ou étrangers, des laboratoires publics ou privés. 


\title{
Dielectric function and optical properties of Al-rich AlInN alloys pseudomorphically grown on GaN
}

\author{
E. Sakalauskas ${ }^{1}$, H. Behmenburg ${ }^{2,3}$, C. Hums ${ }^{4}$, P. Schley ${ }^{1}$, \\ G. Rossbach ${ }^{1}$, C. Giesen ${ }^{2}$, M. Heuken ${ }^{2,3}$, H. Kalisch ${ }^{3}$, \\ R. H. Jansen ${ }^{3}$, J. Bläsing ${ }^{4}$, A. Dadgar ${ }^{4}$, A. Krost ${ }^{4}$, \\ R. Goldhahn ${ }^{1,4}$ \\ ${ }^{1}$ Institut für Physik, Technische Universität Ilmenau, PF 100565, 98684 Ilmenau, \\ Germany \\ 2 AIXTRON AG, Kaiserstr. 98, 52134 Herzogenrath , Germany \\ ${ }^{3}$ Institut für Theoretische Elektrotechnik, RWTH Aachen University, Kackertstr. \\ 15-17, 52072 Aachen, Germany \\ ${ }^{4}$ Institut für Experimentelle Physik, Otto-von-Guericke-Universität Magdeburg, PF \\ 4120, 39016 Magdeburg, Germany \\ E-mail: egidijus.sakalauskas@tu-ilmenau.de
}

\begin{abstract}
A detailed discussion of the optical properties of Al-rich $\mathrm{Al}_{1-x} \operatorname{In}_{x} \mathrm{~N}$ alloy films is presented. The (0001)-oriented layers with In contents between $x=0.143$ and $x=0.242$ were grown by metal-organic vapor phase epitaxy on thick GaN buffers. Sapphire or $\mathrm{Si}(111)$ served as the substrate. High-resolution X-ray diffraction revealed pseudomorphic growth of the nearly lattice-matched alloys; the data analysis yielded the composition as well as the in-plain strain. The complex dielectric function (DF) between 1 and $10 \mathrm{eV}$ was determined from spectroscopic ellipsometry measurements. The sharp onset of the imaginary part of the DF defines the direct absorption edge, while clearly visible features in the high-photon energy range of the DF, attributed to critical points of the band structure, indicate promising crystalline quality of the AlInN layers. It is demonstrated that the experimental data can be well reproduced by an analytical DF model. The extracted characteristic transition energies are used to determine the bowing parameters for all critical points of the band structure. In particular, strain and the high exciton binding energies for the Al-rich alloys are taken into account in order to assess the splitting between the valence band with $\Gamma_{9}^{\mathrm{V}}$ symmetry and the $\Gamma_{7}^{\mathrm{c}}$ conduction band at the center of the Brillouin zone. Finally, the compositional dependence of the high-frequency dielectric constants is reported.
\end{abstract}

PACS numbers: 78.20.Ci, 78.55.Cr, 78.66.Fd, 81.05.Ea, 81.70.Fy

Submitted to: J. Phys. D: Appl. Phys. 


\section{Introduction}

The hexagonal $\mathrm{Al}_{1-x} \operatorname{In}_{x} \mathrm{~N}$ alloy system offers various unique properties which can improve the performance of electronic and optoelectronic devices and extend the application range of group-III nitride semiconductors. Alloys with 17-18 \% indium content can be grown lattice-matched (LM) to GaN, i.e. the thickness of corresponding AlInN films can be varied in a wide range while strain-induced relaxation is avoided. They are used e.g. for AlInN/GaN-based high electron mobility transistors (HEMTs) for high-frequency and high-power microwave applications $[1,2,3,4,5]$. The advantage of these devices is the very high density of the two-dimensional electron gas in the channel layer induced by large spontaneous polarization fields. LM $\mathrm{Al}_{1-x} \mathrm{In}_{x} \mathrm{~N}$ has also a great potential for the application in highly-reflecting distributed Bragg reflectors (DBRs), light-emitting diodes (LEDs), laser diodes (LDs), detectors and sensors [6, 7, 8, 9, 10]. Recently, high reflectivity airgap distributed Bragg reflectors were fabricated by wet eching of LM AlInN sacrificial layers $[11,12,13]$ and microdisk ( $\mu$-disk) technology, which is used for the new advanced devices, was developed [14]. In the work of Watson et al it was demonstrated that the additional insertion of LM AlInN layer into IIInitride multilayer structures grown on free-standing GaN substrate facilitates the $i n$ situ reflectence measurements, which allow accurate controll of layer thicknesses [15]. A comprehensive review of properties and applications of AlInN lattice-matched to GaN has been published elsewhere [16].

Despite the intensive research, many properties of AlInN alloys are not very precisely known over the whole compositional range. The change of the band gap with In content as well as reliable optical functions, such as the complex dielectric function (DF) $\bar{\varepsilon}=\varepsilon_{1}+i \varepsilon_{2}$ or the complex index of refraction $\bar{N}=n+i k=\sqrt{\bar{\varepsilon}}$ to be used for the design of wave guides or DBRs, are two of the open questions. Both are closely related because the valence band (VB) ordering has a strong impact on the optical anisotropy found in hexagonal nitrides. It is known that the splitting between the uppermost VB and lowest conduction band $(\mathrm{CB})$ at the $\Gamma$-point of the Brillouin zone increases at room temperature (RT) from 0.67(5) eV for InN $[17,18]$ to $6.015 \mathrm{eV}$ for $\mathrm{AlN}[19,20]$. However, it is accompanied by a change of the symmetry for the wave function of the highest VB as experimentally evidenced for the two binary compounds in Refs. [21, 22, 23] and $[24,25]$, respectively. The discussion of the ordering for the alloys will be presented below. Furthermore, the exciton binding energies rise with the band gap from $\sim 2 \mathrm{meV}$ [26] to $55 \mathrm{meV}$ [20]. It causes an increasing energy difference between the position of the absorption edge and the VB-CB spacing in the band structure. These effects together with the different methods applied to extract band-gap energies might explain the large spread of bowing parameters [27, 28, 29, 30, 31, 32] reported in recent years.

The very accurate representation of the DF or the complex refractive index by analytical models $[33,34]$ requires experimental data which cover also the vacuumultraviolet (VUV) spectral range because both, the band gap region and contributions from high-energy critical points (CPs) of the band structure (also known as van Hove 
Table 1. Values for the surface roughness and the AlInN layer thickness as determined by AFM, SE, and XRD.

\begin{tabular}{l|cc|cc}
\hline \multirow{2}{*}{ Sample (In content) } & \multicolumn{2}{|c|}{ surface roughness $(\mathrm{nm})$} & \multicolumn{2}{|c}{ alloy thickness $(\mathrm{nm})$} \\
\cline { 2 - 5 } & AFM $(\mathrm{rms})$ & SE & XRD & SE \\
\hline SA1 $(14.3 \%)$ & 1.1 & 2.0 & 72 & 73 \\
SA2 $(17.5 \%)$ & 1.2 & 2.2 & 75 & 74 \\
SA3 $(19.0 \%)$ & 1.4 & 2.4 & 78 & 77 \\
SA4 $(22.8 \%)$ & 1.4 & 3.6 & 80 & 80 \\
SI1 $(17.4 \%)$ & 2.1 & 6.2 & 47 & 48 \\
SI2 $(24.2 \%)$ & 1.4 & 1.9 & 55 & 54 \\
\hline
\end{tabular}

singularities) [35], determine the dispersion of the optical functions. Ellipsometry studies based on synchrotron radiation provide those data which were demonstrated, e.g. for Al-rich AlGaN [36] and for In-rich InAlN [27] and InGaN [37] alloys.

The current paper reports on the determination and interpretation of the DF for Al-rich AlInN alloys. After the presentation of the experimental results, some fundamentals of the band structure (band ordering) and optical selection rules are summarized which are essential for an understanding of the used analytical DF model. It is demonstrated that this model is well suited to describe the whole spectral range. Then, the compositional dependence of the fitted characteristic energies is discussed and compared to the related work. Finally, the dispersion of the real part of the DF below the band gap is analyzed in more detail.

\section{Experimental}

Two sets of $\mathrm{Al}_{1-x} \operatorname{In}_{x} \mathrm{~N}$ samples are investigated. The first set consists of four samples with In contents of 14.3, 17.5, 19.0 and $22.8 \%$ which are labeled as SA1, SA2, SA3, and SA4, respectively. The hexagonal (0001)-oriented $\mathrm{Al}_{1-x} \mathrm{In}_{x} \mathrm{~N}$ films were deposited by metal-organic vapor phase epitaxy (MOVPE) on sapphire substrates employing a 3.5 $\mu \mathrm{m}$ thick GaN buffer layer. The growth temperature was varied from 715 up to $795{ }^{\circ} \mathrm{C}$ leading to a decrease of the In content from 22.8 to $14.3 \%$. The alloy layer thicknesses were determined by spectroscopic ellipsometry (SE) and by X-ray diffraction (XRD). They range between 73 and $80 \mathrm{~nm}$ as summarized in Tab. 1.

The second set of samples employed $\mathrm{Si}(111)$ as the substrate. The MOVPE growth started with a AlN seed layer, high-temperature AlN layer, GaN buffer layer, lowtemperature AlN layer for strain compensation, and followed by a final $900 \mathrm{~nm}$ thick GaN buffer as sketched in Fig. 1. This sequence prevents cracking despite the mismatch of the thermal expansion coefficients of the substrate and the nitrides. Results for two samples with In contents of 17.4 and $24.2 \%$ which are denoted by SI1 and SI2, respectively, are reported here. The growth conditions of the alloys are described in detail in Ref. [38]; the layer thicknesses amount to 48 and $54 \mathrm{~nm}$, as determined by SE. 


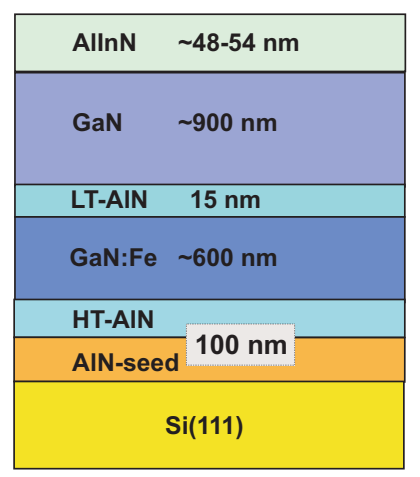

Figure 1. Schematic layer structure of AlInN/Si(111) sample structure.

The structural and surface properties of the films were examined by high-resolution X-ray diffraction (HRXRD) and atomic force microscopy (AFM), respectively.

The SE measurements were carried out in the photon energy range from 1 to $10 \mathrm{eV}$ by using a commercial rotating analyzer ellipsometer and the ellipsometer attached to the Berlin electron storage ring for synchrotron radiation (BESSY II) [39]. The SE spectra in the range from 1 to $6.4 \mathrm{eV}$ were recorded with a resolution of $10 \mathrm{meV}$ and at different angles of incidence $\left(60^{\circ}, 67^{\circ}\right.$, and $\left.74^{\circ}\right)$ in order to reduce the correlation between the optical constants and the layer thickness. The high photon flux provided by the synchrotron radiation allowed to set the spectral resolution to $5 \mathrm{meV}$ in the range above $5.5 \mathrm{eV}$; the angle of incidence is fixed to $67.5^{\circ}$.

Finally, photoluminescence (PL) measurements were conducted at RT by using $5^{\text {th }}$ harmonic of Nd:YAG laser with $\lambda_{\text {exc }}=213 \mathrm{~nm}$ and $I_{\text {exc }}=1.7 \mathrm{MW} / \mathrm{cm}^{2}$.

\section{Experimental results}

\subsection{Structural characterization}

The AFM studies revealed for both set of samples a smooth surface morphology which is essential for the accuracy of the SE results. Scans of $5 \times 5 \mu \mathrm{m}^{2}$ area for the first set of samples and $10 \times 10 \mu \mathrm{m}^{2}$ for the second set of samples yielded the root-mean square ( $\mathrm{rms}$ ) roughness, the values are summarized in Tab. 1. They range typically between 1.1 and $1.4 \mathrm{~nm}$, only sample SI1 showed a slightly higher value of $2.1 \mathrm{~nm}$ which is not critical for the optical studies.

The recent studies of hexagonal AlN films deposited on sapphire substrate demonstrated the strong influence of strain on the absorption-related properties around the band gap of the compound [25]. Similar behavior is expected for Al-rich AlInN alloys. Detailed XRD measurements (symmetric diffraction of (0002) planes and grazing incidence in-plane diffraction of (10-10) planes) were therefore carried out in order to determine the lattice constants and mosaicity of both, the GaN buffer layer and the alloy film. Experimental and simulated data of symmetric diffraction (0002) are represented in Fig. 2. 


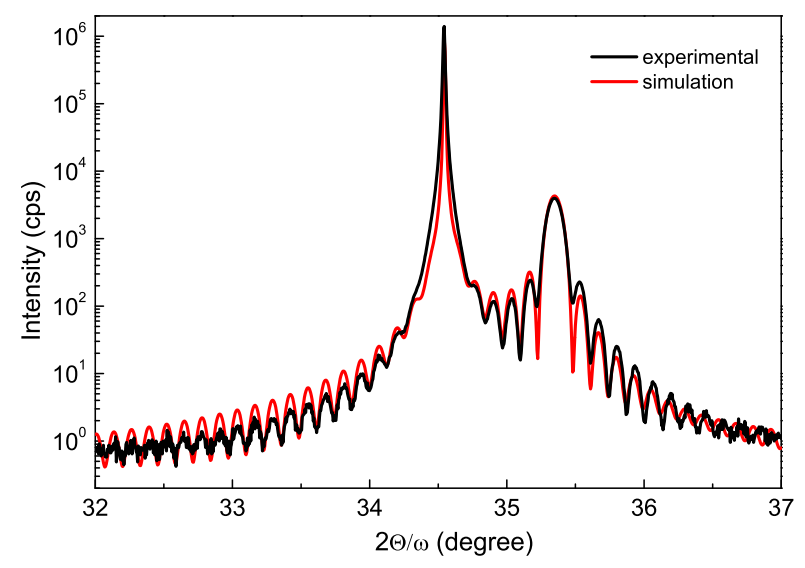

Figure 2. Symmetric X-ray diffraction (0002) and simulation for sample SA1.

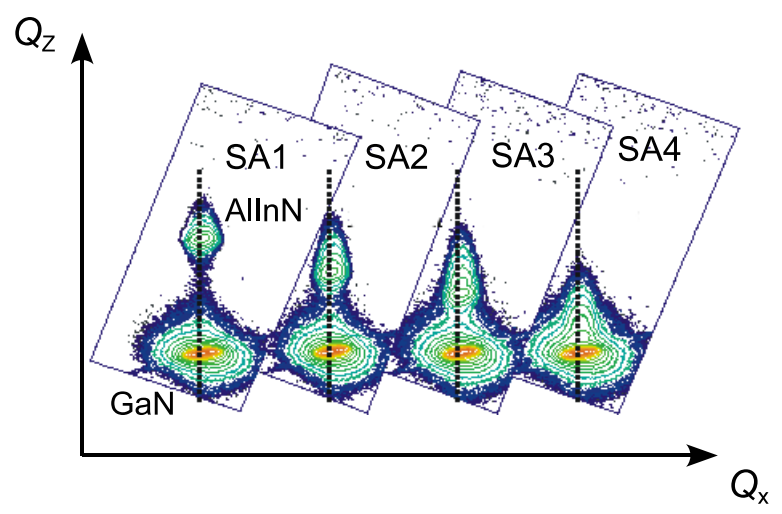

Figure 3. Reciprocal space maps of the (10-15) reflection for samples SA1, SA2, SA3 and SA4.

Figure 3 shows the reciprocal space maps of the (10-15) reflection obtained from XRD analysis for the first set of samples grown on sapphire substrates. AlInN and GaN peaks are aligned in the $Q_{z}$ axis, indicating that AlInN is lattice-matched to the GaN buffer layer. Similar results are found for the samples on $\mathrm{Si}(111)$.

The most critical step of the XRD analysis is the evaluation of the strain in the films and the determination of the composition. Darakchieva et al [40] presented for Al-rich alloys a detailed comparison of XRD data with measurements of the In content by Rutherford backscattering (RBS). The maximum deviation $x_{\mathrm{XRD}}-x_{\mathrm{RBS}}$ amounted to 0.014 if Vegard's law was applied, i.e. there is a tendency for a slight overestimation of the In content by XRD. The use of bowing parameters for the lattice constants [41] reduced the difference only by 0.004 . In this work, Vegard's law is used to determine the composition.

We used the strain-free lattice constants for InN [42], GaN [43], and AlN [44] of $a_{0}^{\mathrm{InN}}=3.5377 \AA$ and $c_{0}^{\mathrm{InN}}=5.7037 \AA, a_{0}^{\mathrm{GaN}}=3.1893 \AA$ and $c_{0}^{\mathrm{GaN}}=5.1852 \AA$ as well as $a_{0}^{\mathrm{AlN}}=3.112 \AA$ and $c_{0}^{\mathrm{AlN}}=4.9809 \AA$, respectively. The analysis starts from the characterization of the buffer layers. GaN lattice parameters of $a=3.183 \AA$ and $c=5.189 \AA$ 
are found for the films on sapphire indicating slight compressive in-plane strain which is typical for the growth on this substrate. Mosaic twist and tilt amount to less than $0.1^{\circ}$ emphasizing the good quality of the layers. The films on $\mathrm{Si}(111)$ exhibit in contrast a weak tensile strain as indicated by the lattice parameters of $a=3.193 \AA$ and $c=5.182 \AA$. Applying the different $a$ values for the pseudomorphically grown alloys, the strained $c^{\mathrm{AlInN}}$ lattice parameters as function of $x$ were calculated and compared to the experimental results yielding finally the composition. As in Ref. [40], the stiffness constants $C_{13}$ and $C_{33}$ of $\mathrm{InN}$ and AlN were taken from Ref. [45], the values for the alloys were obtained by linear interpolation.

\subsection{Dielectric function}

The hexagonal group-III nitrides are optically anisotropic materials for which at least two tensor components differ from each other. Strain-free wurtzite crystals are uniaxial materials with respect to the polar $\boldsymbol{c}$-axis. The ordinary $\left(\bar{\varepsilon}_{\mathrm{o}}\right)$ and extraordinary $\left(\bar{\varepsilon}_{\mathrm{e}}\right)$ dielectric tensor components express the optical response for electric-field polarization (E) perpendicular $(\boldsymbol{E} \perp \boldsymbol{c})$ and parallel $(\boldsymbol{E} \| \boldsymbol{c})$ to the optical axis, respectively.

For the (0001)-oriented films studied here, the in-plane strain is isotropic which means that the anisotropy remains uniaxial. However, although SE measures the optical response under a certain angle of incidence, the ellipsometric parameters $\Psi$ and $\Delta$ reflect mainly the spectral dependence of the ordinary DF attributed to the high index of refraction. Noteworthy deviations around the band gap are only found if the material exhibits sharp excitonic resonances for both polarization directions [25, 46]. This does not apply here due to the alloy broadening. In the range of the high-energy critical points (van Hove singularities) only a minor enhancement of the magnitude in comparison to the true ordinary DF might be expected which was evidenced by comparing the data for (0001)- and (11) 0 )-oriented InN films [21].

The complex DF was obtained by fitting the ellipsometric parameters $\Psi$ and $\Delta$ using a multi-layer model [47]. No assumption was made concerning the spectral shape of DF, i.e. $\varepsilon_{1}$ and $\varepsilon_{2}$ were separately fitted for each photon energy followed by a proof of Kramers-Kronig consistency between both quantities. Surface roughness affects the spectroscopic ellipsometry data. Thus, it is important to account for it in the multilayer model. The surface roughness was modeled using the Bruggeman effective medium approximation assuming $50 \%$ air voids in AlInN matrix. This overlayer thickness was also fitted. Consistent with previous studies (see the detailed discussion in Ref. [48]), slightly larger values are determined by the optical method in comparison to the AFM data in Table 1.

The obtained dielectric functions of samples SA1, SA2, SA3, and SA4 in the spectral range from 1 to $10 \mathrm{eV}$ are displayed in Fig. 4. Pronounced features attributed to critical points of the band structure are found in the high-energy range of $\varepsilon_{2}$. They have a strong impact on the dispersion of $\varepsilon_{1}$ at lower energies. For the second set of samples (AlInN/Si(111)), data are only available so far for the range from 1 to $6.42 \mathrm{eV}$. 
Figure 5 (a) and (b) shows the results around the band gap for the AlInN/sapphire and AlInN/Si(111) samples, respectively. It is clearly seen that with increasing In content, the absorption onset (imaginary part of the dielectric function $-\varepsilon_{2}$ ) redshifts. Following the Kramers-Kronig relation, the $\varepsilon_{1}$ peak also shifts to the lower energies. Fig. 5 (b) also shows the DF of AlN for comparison.

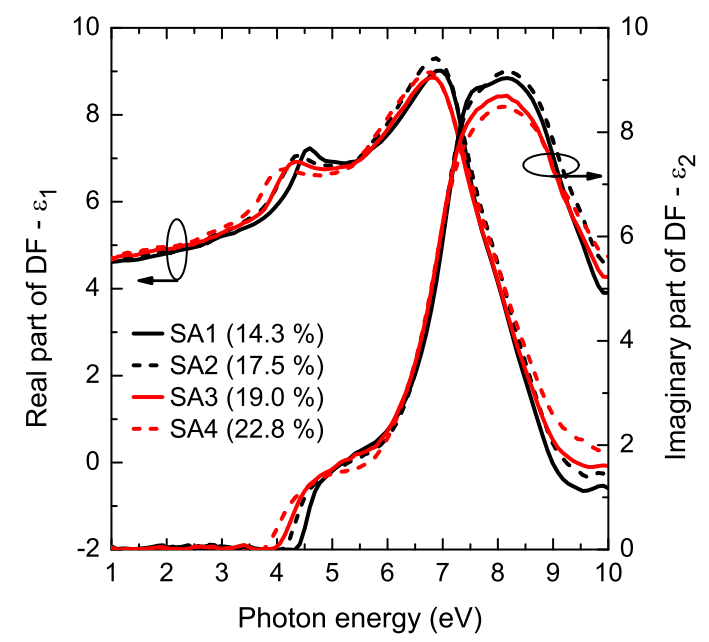

Figure 4. Dielectric functions of the AlInN layers grown sapphire substrate.
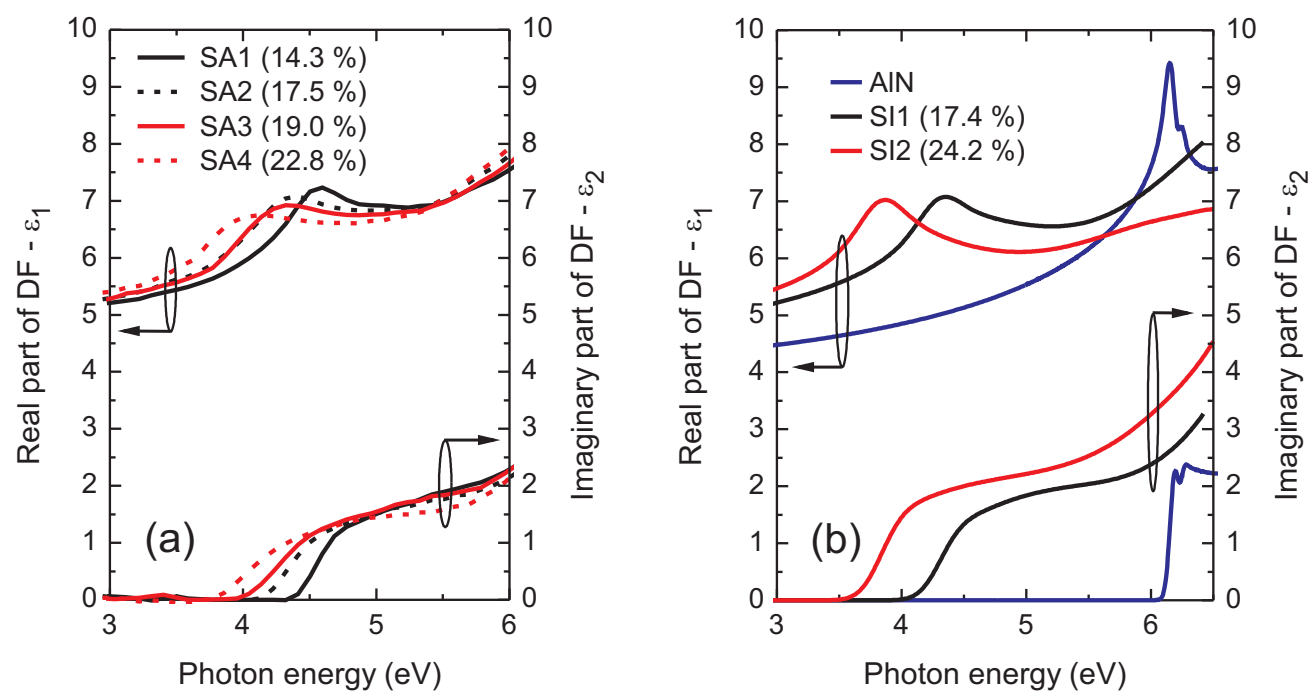

Figure 5. Dielectric function of AlInN around the band gap: for AlInN/sapphire samples (a) and AlInN/Si(111) samples together with data for AlN (b). DF of AlN is taken from Ref. [25] 


\section{Discussion}

\subsection{Band structure and optical selection rules for AlInN alloys}

It is essential to consider the optical selection rules prior to the interpretation of the DF. It starts from the $\mathrm{VB}$ ordering around the $\Gamma$ point of the Brillouin zone and the symmetry of the wave functions. Crystal field $\left(\Delta_{\text {cf }}\right)$ and spin-orbit interaction $\left(\Delta_{\text {so }}\right)$ split the VB maximum into three two-fold-degenerate VBs with $\Gamma_{9}^{\mathrm{v}}, \Gamma_{7-}^{\mathrm{v}}$, and $\Gamma_{7+}^{\mathrm{v}}$ symmetry. All hexagonal nitrides are direct band-gap materials with absolute CB minimum denoted by $\Gamma_{7}^{\mathrm{c}}$. According to the quasi-cubic model [49], the energetic position of the bands is given by

$$
\begin{aligned}
& \Gamma_{7}^{\mathrm{c}}=\Delta_{\mathrm{cf}}+\Delta_{\mathrm{so}} / 3+E_{\mathrm{A}} \\
& \Gamma_{9}^{\mathrm{v}}=\Delta_{\mathrm{cf}}+\Delta_{\mathrm{so}} / 3 \\
& \Gamma_{7+}^{\mathrm{v}}=\Delta_{\mathrm{cf}} / 2-\Delta_{\mathrm{so}} / 6+\frac{1}{2} \sqrt{\left(\Delta_{\mathrm{cf}}+\Delta_{\mathrm{so}}\right)^{2}-\frac{8}{3} \Delta_{\mathrm{cf}} \Delta_{\mathrm{so}}} \\
& \Gamma_{7-}^{\mathrm{v}}=\Delta_{\mathrm{cf}} / 2-\Delta_{\mathrm{so}} / 6-\frac{1}{2} \sqrt{\left(\Delta_{\mathrm{cf}}+\Delta_{\mathrm{so}}\right)^{2}-\frac{8}{3} \Delta_{\mathrm{cf}} \Delta_{\mathrm{so}}} .
\end{aligned}
$$

The energy difference $\Gamma_{7}^{\mathrm{c}}-\Gamma_{9}^{\mathrm{v}}=E_{\mathrm{A}}$ for strain free material is always used as reference point for the analysis of interband absorption. This definition is essential when considering the compositional dependence of transition energies. InN has a spin-orbit energy of $5 \mathrm{meV}$ and crystal-field splitting energy of $19 \mathrm{meV}$ [50]. AlN has a slightly larger spin-orbit energy of $20 \mathrm{meV}$ and a large negative crystal-field splitting energy of $-230 \mathrm{meV}[24]$. It is worth pointing out that the sign of crystal field energy inverts the valence band ordering as shown in Fig. 6 .

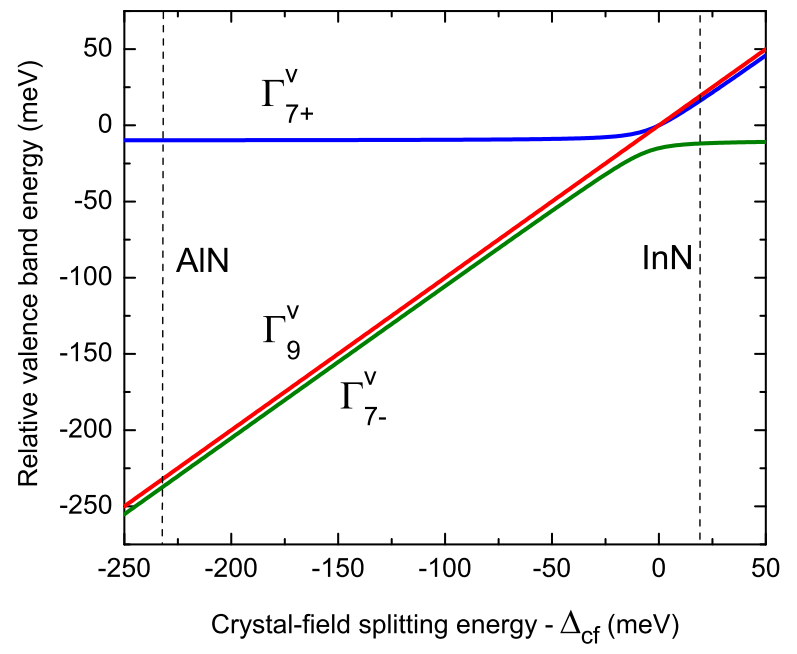

Figure 6. Valence band ordering as a function of crystal-field splitting energy for the hexagonal AlInN alloy system.

Therefore, the band crossing will occur for $\mathrm{Al}_{1-x} \operatorname{In}_{x} \mathrm{~N}$ alloy at $\Delta_{\text {cf }}=0 \mathrm{eV}$ (approximately at $x=0.92$, if a linear crystal-field splitting energy interpolation is used). 
No studies have been reported so far considering the change of $\Delta_{\text {cf }}$ with the In content.

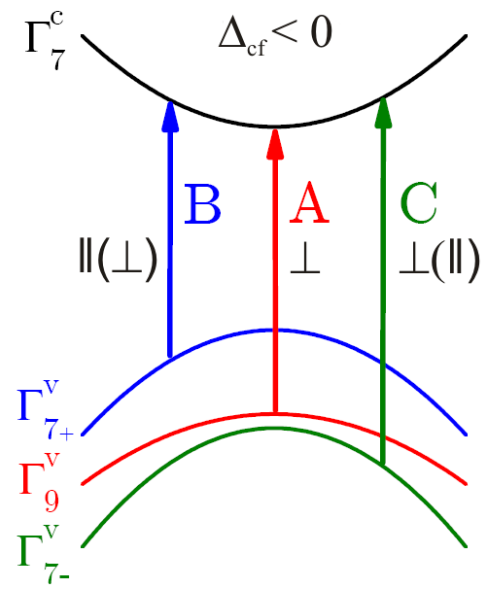

Figure 7. Simplified scheme of transitions A, B, and C (for $\Delta_{\text {cf }}<0$ ). Symbols $\perp$ and $\|$ indicate strong transition probability, meanwhile symbols $(\perp)$ and $(\|)$ indicate weak transition probability for the respective polarization states.

For Al-rich AlInN the transitions from $\Gamma_{9}^{\mathrm{v}}$ valence band to the $\Gamma_{7}^{\mathrm{c}}$ conduction band (labeled A) and from $\Gamma_{7-}^{\mathrm{v}}$ to $\Gamma_{7}^{\mathrm{c}}$ (labeled C) exhibit strong relative oscillator strengths only for the configuration $\boldsymbol{E} \perp \boldsymbol{c}$. Thus by measuring c-plane InAlN samples with an ellipsometer (almost $\boldsymbol{E} \perp \boldsymbol{c}$ ), these transitions and the corresponding free excitonic transitions $E_{\mathrm{FX}}$ and $E_{\mathrm{FXC}}$ dominate the optical response. In contrast, the transition from $\Gamma_{7+}^{\mathrm{v}}$ to $\Gamma_{7}^{\mathrm{c}}$ (labeled B) is strongly allowed only for the configuration $\boldsymbol{E} \| \boldsymbol{c}$. The simplified valence band ordering scheme sketched in Fig. 7 shows that transition B stems from the topmost valence band, but its oscillator strength is weak. It leads to the fact that it is hardly detectable in the ordinary DF and only weakly observable in PL studies. A detailed discussion of the selection rules for nitrides and their implications on the analysis of optical studies can be found in Ref. [33].

\subsection{Analytical dielectric function representation}

Accurate dielectric function representation in analytical form is useful for analysis of material optical properties and also needed for device modeling. A detailed description of the DF model used here can be found in Ref. [33]. In short, the imaginary part of the DF is described by the following expression:

$$
\varepsilon_{2}=\varepsilon_{2, \text { low }}+\varepsilon_{2, \text { high }} .
$$

Here the term $\varepsilon_{2, \text { low }}$ describes $\varepsilon_{2}$ in the band gap region and the second term $\varepsilon_{2, \text { high }}$ - in higher energy range. They are expressed as follows:

$$
\begin{aligned}
\varepsilon_{2, \text { low }}= & \sum_{\mathrm{j}=\mathrm{A}, \mathrm{C}} \frac{A_{\mathrm{BS}} \hbar \omega \Gamma_{\mathrm{BS}}}{\left(\left(E_{\mathrm{j}}-R\right)^{2}-\hbar^{2} \omega^{2}\right)+\left(\hbar \Gamma_{\mathrm{BS}}\right)^{2}} \\
& +\sum_{\mathrm{j}=\mathrm{A}, \mathrm{C}} \frac{A_{\mathrm{CS}}}{\hbar \omega} \frac{1+\operatorname{erf}\left[\left(\hbar \omega-E_{\mathrm{j}}\right) / \Gamma_{\mathrm{CS}}\right]}{1-\exp \left(-2 \pi \sqrt{R /\left|\hbar \omega-E_{\mathrm{j}}\right|}\right)} ;
\end{aligned}
$$




$$
\begin{aligned}
\varepsilon_{2, \text { high }}= & \sum_{\mathrm{j}=1,2,3} \frac{A_{\mathrm{j}} \hbar \omega \Gamma_{\mathrm{j}}}{\left(E_{\mathrm{j}}^{2}-\hbar^{2} \omega^{2}\right)^{2}+\left(\hbar \omega \Gamma_{\mathrm{j}}\right)^{2}} \times \\
& \times\left[\Theta\left(E_{\mathrm{j}}-\hbar \omega\right) \frac{\hbar \omega-E_{\mathrm{A}}}{E_{\mathrm{j}}-E_{\mathrm{A}}}+\Theta\left(\hbar \omega-E_{\mathrm{j}}\right)\right] .
\end{aligned}
$$

The first term in $\varepsilon_{2, \text { low }}(6)$ expressed by the real part of damped harmonic oscillators (DHO) represents the bound exciton states (BS) below the band gap, meanwhile the second term represents the exciton continuum. The high energy critical points (CPs) are described by DHO's with the Heaviside step function $\Theta$ in the Eq. 7.

The real part of the DF can be described by:

$$
\begin{aligned}
\varepsilon_{1}= & +\sum_{\mathrm{j}=\mathrm{A}, \mathrm{C}}\left(\frac{A_{\mathrm{BS}}\left(\left(E_{\mathrm{j}}-R\right)^{2}-\hbar^{2} \omega^{2}\right)}{\left(\left(E_{\mathrm{j}}-R\right)^{2}-\hbar^{2} \omega^{2}\right)^{2}+\left(\hbar \omega \Gamma_{\mathrm{BS}}\right)^{2}}\right) \\
& +\frac{1}{\pi} \Re\left(A_{0} \ln \frac{E_{\mathrm{P}}^{2}-\left(\hbar \omega+\mathrm{i} \Gamma_{0}\right)^{2}}{E_{\mathrm{A}}^{2}-\left(\hbar \omega+\mathrm{i} \Gamma_{0}\right)^{2}}\right) \\
& +\frac{1}{\pi} \Re\left(\frac{A_{\mathrm{P}} E_{\mathrm{P}}}{E_{\mathrm{P}}^{2}-\left(\hbar \omega+\mathrm{i} \Gamma_{0}\right)^{2}}\right) \\
& +\sum_{\mathrm{j}=1,2,3} \frac{A_{\mathrm{j}}\left(E_{\mathrm{j}}^{2}-\hbar^{2} \omega^{2}\right)}{\left(E_{\mathrm{j}}^{2}-\hbar^{2} \omega^{2}\right)^{2}+\left(\hbar \omega \Gamma_{\mathrm{j}}\right)^{2}} .
\end{aligned}
$$

The DHOs in the Eq. 8 represent the bound excitonic states and high energy CPs, the excitonic continuum is modeled by a logarithmic function and $b$ is a constant term.

Experimentally obtained real and imaginary parts of the dielectric function were fitted simultaneously with analytical expressions (Eq. 5 and Eq. 8) using the least square method. The fitting parameters are listed in Tab. 2. The experimental and calculated (from the analytical model) complex dielectric function and complex index of refraction for sample SA1 are represented in Fig. 8 (a) and (b), respectively.
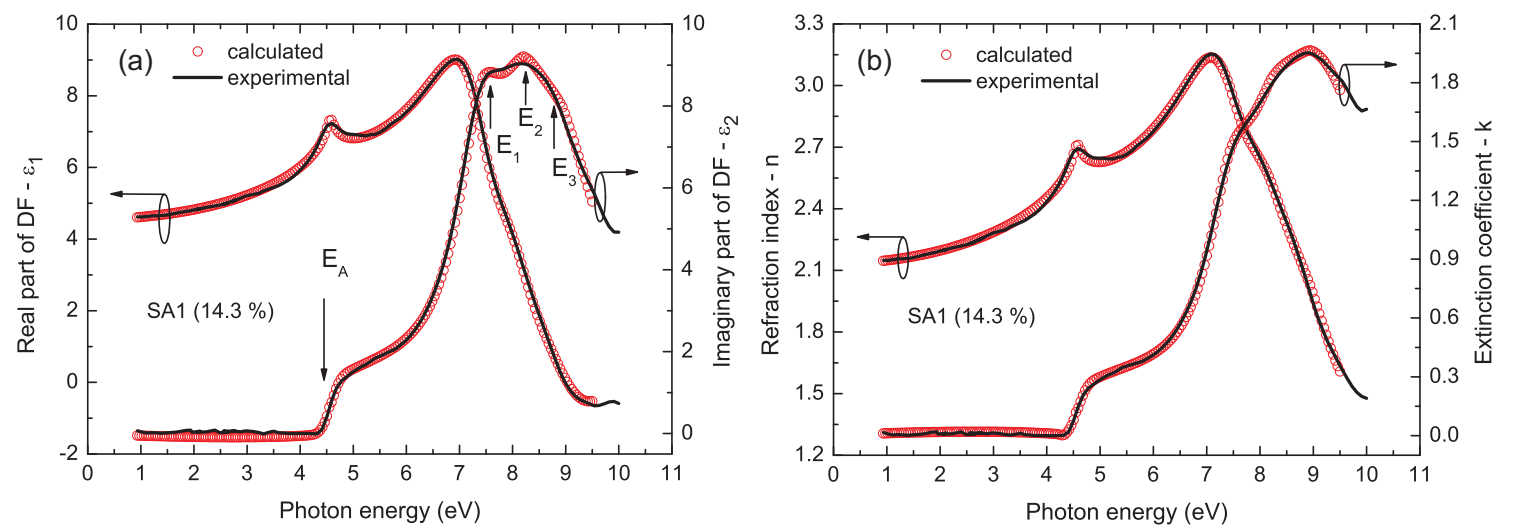

Figure 8. Experimental and calculated complex DF (a) and complex index of refraction (b) of sample SA1 (In content $14.3 \%$ ).

The difference between transition energy $E_{\mathrm{A}}$ and $E_{\mathrm{C}}$ was kept fixed at $13 \mathrm{meV}$, which corresponds to the linearly interpolated spin-orbit splitting energy at $x=0.18$. 
From the fitted data, we obtained the exciton binding energy to be 29, 31, 35, and $17 \mathrm{meV}$ for samples SA1, SA2, SA3, and SA4, respectively. The exciton energy value for sample SA4 differs strongly from the values obtained for samples SA1, SA2, and SA3. In comparison with other samples, sample SA4 also has the broadest absorption onset and the critical point peaks are not so sharp as is seen in Fig. 4, which indicates its rather poor crystalline quality. Therefore, we ignore the exciton energy value obtained for sample SA4. We estimate the exciton binding energy to be about $30 \mathrm{meV}$, which is close to the values obtained for the samples SA1, SA2, and SA3.

The critical point energies obtained by fitting the analytical DF model to experimental data are in good agreement with the energies determined from the third derivative method.

Critical points of the band structure were also determined by calculating the third derivative of the point-by-point DF multiplied by the square of the photon energy $(E)$ and fitting the resulting spectra using expression [51]:

$$
\frac{d^{3}}{d E^{3}}\left(E^{2} \bar{\varepsilon}\right)=\sum_{\mathrm{j}=1}^{\mathrm{N}} e^{\mathrm{i} \phi_{\mathrm{j}}} \frac{C_{\mathrm{j}}}{\left(E+i \Gamma_{\mathrm{j}}-E_{\mathrm{g}, \mathrm{j}}\right)^{n / 2}},
$$

where $\phi_{\mathrm{j}}, C_{\mathrm{j}}, \Gamma_{\mathrm{j}}$, and $E_{\mathrm{g}, \mathrm{j}}$ denote the phase angle, the magnitude, the broadening energy, and the transition energy of the $\mathrm{j}$-th $\mathrm{CP}$, respectively.

The critical point energies determined by the third derivative method are summarized in Tab. 3 for the AlInN/sapphire sample set. SE measurements for the AlInN/Si(111) sample set were conducted only around the band gap. The transition energy $E_{\mathrm{A}}$ (transition from $\Gamma_{9}^{\mathrm{v}}$ valence band to the $\Gamma_{7}^{\mathrm{c}}$ conduction band) values of 4.34 and $3.85 \mathrm{eV}$ were determined for samples SI1 and SI2, respectively.

\subsection{Strain influence on the band gap energy}

The strain influence on the band gap must be accounted for before evaluating the bowing parameter for the AlInN alloy. A six-band $\mathbf{k} \cdot \mathbf{p}$ model [52] is used to calculate the strain influence.

All $\mathrm{Al}_{1-\mathrm{x}} \mathrm{In}_{\mathrm{x}} \mathrm{N}$ parameters (spin-orbit, crystal-field splitting energy, deformation potentials, and elastic stiffness constants) are deduced from InN and AlN parameters by using a linear interpolation. The deformation potentials $\left(a_{c z}-D_{1}, a_{c t}-D_{2}, D_{3}, D_{4}\right.$, and $\left.D_{5}\right)$ were taken from Ref. [53] and deformation potentials $a_{c z}=a_{c t}$ from Ref. [54] for AlN and InN. Elastic constants $C_{13}$ and $C_{33}$ were employed from Ref. [55] for AlN and InN. Crystal-field splitting energy $\left(\Delta_{\text {cf }}\right)$ and spin-orbit energy $\left(\Delta_{\text {so }}\right)$ were used from Ref. [24] (AlN) and from Ref. [50] (InN).

For the (0001)-oriented films studied here, the in-plane strain is isotropic $\left(\epsilon_{x x}=\epsilon_{y y}\right)$ and is expressed as:

$$
\epsilon_{x x}=\epsilon_{y y}=\frac{a-a_{\mathrm{relax}}^{\mathrm{AlInN}}}{a_{\mathrm{relax}}^{\mathrm{AlnN}}},
$$


where $a$ is in-plane lattice parameter of strained AlInN (identical to the respective GaN buffers, see subsection 3.1). The out-of-plane strain $\epsilon_{z z}$ is described as follows:

$$
\epsilon_{z z}=-2 \frac{C_{13}^{\mathrm{AlInN}}}{C_{33}^{\mathrm{AlInN}}} \epsilon_{x x}
$$

The lattice parameter of unstrained $a_{\mathrm{relax}}^{\mathrm{AlInN}}$ was derived by applying Vegard's law:

$$
a_{\mathrm{relax}}^{\mathrm{AlInN}}=a^{\mathrm{InN}} x+a^{\mathrm{AlN}}(1-x) .
$$

As already mentioned, the elastic stiffness constants $C_{13}^{\mathrm{AlInN}}$ and $C_{33}^{\mathrm{AlInN}}$ for $\mathrm{Al}_{1-x} \mathrm{In}_{x} \mathrm{~N}$ were also interpolated linearly.

The strain-induced energy shifts $\Delta E_{\mathrm{A}}^{\text {strain }}$ calculated from the $\mathbf{k} \cdot \mathbf{p}$ model around the $\Gamma$ point of the Brillouin zone, transition energies $E_{\mathrm{A}}$ obtained from DF (influenced by strain), and derived unstrained transition energies $E_{\mathrm{A}}^{\text {relax }}=E_{\mathrm{A}}-\Delta E_{\mathrm{A}}^{\text {strain }}$ values are summarized in Table 4. The following should be noticed. Pseudomorphic AlInN films with low In-content experience tensile strain leading to a red-shift of the experimental data for the $E_{A}$ transition. For high In-content, a strain-induced blue shift up to

Table 2. Fitting parameters for the DF analytical model.

\begin{tabular}{lcccc}
\hline Sample & SA1 $(14.3 \%)$ & SA2 $(17.5 \%)$ & SA3 $(19.0 \%)$ & SA4 $(22.8 \%)$ \\
\hline$A_{0}$ & 1.24 & 1.08 & 1.05 & 0.99 \\
$A_{1}$ & 44.85 & 43.63 & 42.74 & 43.31 \\
$A_{2}$ & 37.01 & 50.13 & 47.84 & 55.82 \\
$A_{3}$ & 70.76 & 79.52 & 74.84 & 82.98 \\
$A_{\mathrm{BS}}$ & $5.8 \cdot 10^{-3}$ & $1.5 \cdot 10^{-2}$ & $9.2 \cdot 10^{-3}$ & 0.46 \\
$A_{\mathrm{CS}}$ & 1.5 & 1.26 & 1.33 & 0.78 \\
$A_{\mathrm{P}}$ & 158.05 & 189.02 & 172.88 & 270.09 \\
$E_{1}(\mathrm{eV})$ & 7.42 & 7.37 & 7.33 & 7.30 \\
$E_{2}(\mathrm{eV})$ & 8.20 & 8.20 & 8.17 & 8.20 \\
$E_{3}(\mathrm{eV})$ & 8.94 & 9.06 & 9.07 & 9.21 \\
$E_{\mathrm{A}}(\mathrm{eV})$ & 4.57 & 4.35 & 4.30 & 4.04 \\
$E_{\mathrm{C}}(\mathrm{eV})$ & $E_{A}+0.013$ & $E_{A}+0.013$ & $E_{A}+0.013$ & $E_{A}+0.013$ \\
$E_{\mathrm{P}}(\mathrm{eV})$ & 15.54 & 15.33 & 15.45 & 16.27 \\
$\Gamma_{0}(\mathrm{eV})$ & $4.4 \cdot 10^{-2}$ & $4.4 \cdot 10^{-2}$ & $5.2 \cdot 10^{-2}$ & $5.4 \cdot 10^{-2}$ \\
$\Gamma_{1}(\mathrm{eV})$ & 1.21 & 1.27 & 1.33 & 1.41 \\
$\Gamma_{2}(\mathrm{eV})$ & 1.31 & 1.47 & 1.52 & 1.65 \\
$\Gamma_{3}(\mathrm{eV})$ & 1.89 & 2.07 & 2.15 & 2.33 \\
$\Gamma_{\mathrm{BS}}(\mathrm{eV})$ & $3.3 \cdot 10^{-2}$ & 0.13 & 0.13 & 2.78 \\
$\Gamma_{\mathrm{CS}}(\mathrm{eV})$ & 0.1824 & 0.20 & 0.28 & 0.19 \\
$R(\mathrm{meV})$ & 29 & 31 & 35 & 17 \\
$b$ & -1.9 & -2.7 & -2.2 & -4.18 \\
\hline & & & &
\end{tabular}


Table 3. High-energy critical point energies for AlInN/sapphire samples.

\begin{tabular}{lccc}
\hline Sample & $\begin{array}{c}E_{1} \\
(\mathrm{eV})\end{array}$ & $\begin{array}{c}E_{2} \\
(\mathrm{eV})\end{array}$ & $\begin{array}{c}E_{3} \\
(\mathrm{eV})\end{array}$ \\
\hline SA1 $(14.3 \%)$ & 7.40 & 8.11 & 8.81 \\
SA2 $(17.5 \%)$ & 7.31 & 8.15 & 8.80 \\
SA3 $(19.0 \%)$ & 7.28 & 8.12 & 8.80 \\
SA4 $(22.8 \%)$ & 7.18 & 8.08 & 8.79 \\
\hline
\end{tabular}

$\sim-140 \mathrm{meV}$ is found for the layers studied here. It is obvious that this effect has to be taken into account for determining bowing parameters for the band gap.

Table 4. Calculated strain-free transition energy $E_{\mathrm{A}}^{\text {relax }}$

\begin{tabular}{lccc}
\hline Sample & $\begin{array}{c}E_{\mathrm{A}} \\
(\mathrm{eV})\end{array}$ & $\begin{array}{c}\Delta E_{\mathrm{A}}^{\text {strain }} \\
(\mathrm{eV})\end{array}$ & $\begin{array}{c}E_{\mathrm{A}}^{\text {relax }} \\
(\mathrm{eV})\end{array}$ \\
\hline SA1 $(14.3 \%)$ & 4.57 & -0.06 & 4.63 \\
SA2 $(17.5 \%)$ & 4.35 & 0.02 & 4.33 \\
SA3 $(19.0 \%)$ & 4.30 & 0.05 & 4.25 \\
SA4 $(22.8 \%)$ & 4.04 & 0.14 & 3.90 \\
SI1 $(17.4 \%)$ & 4.34 & -0.04 & 4.38 \\
SI1 $(24.2 \%)$ & 3.85 & 0.12 & 3.73 \\
\hline
\end{tabular}

\subsection{Bowing parameter}

For accurate band gap (transition $E_{\mathrm{A}}=\Gamma_{7}^{\mathrm{c}}-\Gamma_{9}^{\mathrm{v}}$ ) bowing parameter evaluation we need more experimental data in a wider In content $(x)$ range. Additionally, we employed the data determined by SE from the work of Goldhahn et al [27] for the In-rich AlInN alloys and AlN. Wang et al have evaluated the transition energies for AlInN by PLE [30] which are in good agreement with our values obtained by SE.

The evaluated critical point energy values $E_{1}, E_{2}, E_{3}$ and the band gap energy values $E_{\mathrm{A}}$ determined by $\mathrm{SE}$ ellipsometry for AlInN samples together with the results obtained for AlN, InN and In-rich AlInN alloys [27] are represented in Fig. 9. The fitted bowing parameter $(b)$ of the transition energy $E_{\mathrm{A}}$ is $5.36 \pm 0.36 \mathrm{eV}$ (with endpoint energies of $E^{\mathrm{InN}}=0.675 \mathrm{eV}$ for $\operatorname{InN}$ [18] and $E^{\mathrm{AlN}}=6.21 \mathrm{eV}$ [33] for AlN), which is consistent with the value of $5.3 \mathrm{eV}$ obtained in Ref. [31] and the value of $4.96 \pm 0.28 \mathrm{eV}$ obtained in Ref. [28] within the error interval. The bowing parameters of the highenergy critical points $E_{1}$ and $E_{2}$ were found to be $1.59 \pm 0.07 \mathrm{eV}$ and $2.52 \pm 0.26 \mathrm{eV}$, respectively. For comparison, the transition energy $E_{\mathrm{A}}$ values obtained by modeling optical absorption data for the high quality AlInN samples in the work of Jones et al [29] are represented with the open circles in Fig. 9. Inter-band transition energies as a function of In content $(x)$ together with the fitted bowing curves are also represented in 
Fig. 9.

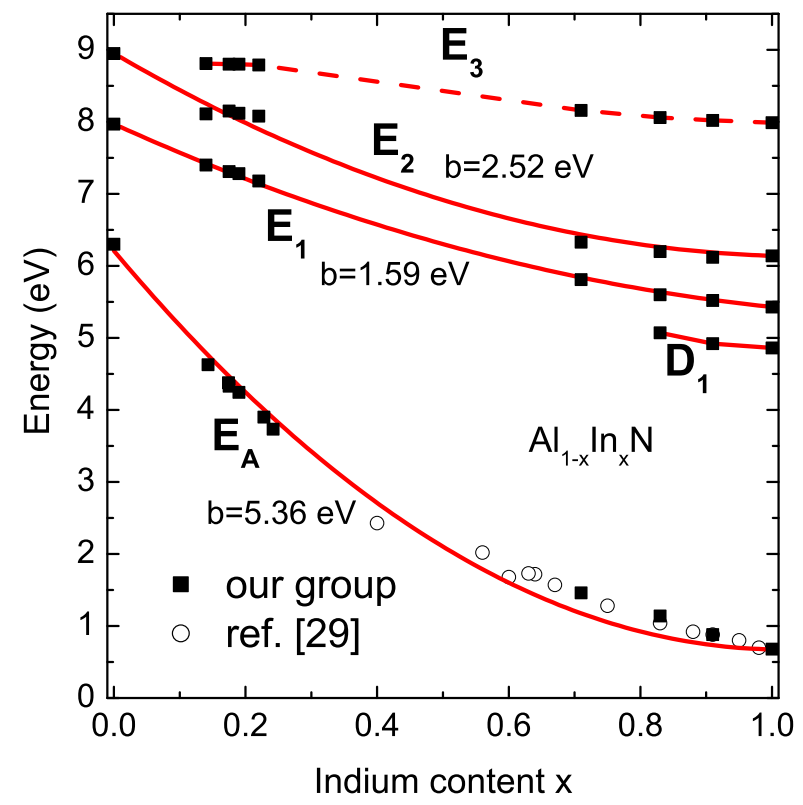

Figure 9. Critical point energies of $\mathrm{Al}_{1-x} \operatorname{In}_{x} \mathrm{~N}$ alloy as a function of In content $(x)$. The red (grey) solid curves represent the inter-band transition energies with the indicated bowing values.

If we look more accurately at the $E_{\mathrm{A}}$ bowing curve (see Fig. 9), we can observe that the fitted curve does not describe well the experimental data points. Therefore, we propose a new empirical expression, which is described as:

$$
E_{\mathrm{j}}^{\mathrm{AlInN}}=E_{\mathrm{j}}^{\mathrm{InN}} \cdot x+E_{\mathrm{j}}^{\mathrm{AlN}} \cdot(1-x)-b_{2}(x) \cdot(1-x) \cdot x,
$$

where index $\mathrm{j}=\mathrm{A}$ and the bowing parameter $b_{2}(x)$ is expressed as a non-linear function of In content $(x)$ :

$$
b_{2}(x)=\frac{A}{1+C \cdot x^{2}} .
$$

The fitted Eq. 13 to the experimental data points yielded the values $\mathrm{A}=6.43 \pm 0.12 \mathrm{eV}$ and $\mathrm{C}=1.21 \pm 0.14$ with the chosen end points $E_{\mathrm{A}}^{\mathrm{InN}}=0.675 \mathrm{eV}$ [18] and $E_{\mathrm{A}}^{\mathrm{AlN}}=6.21 \mathrm{eV}$ [33]. The empirical expression of bowing parameter as a function of In content permits to describe the experimental data points with a smaller error.

Additionally, the PL measurements were conducted on pseudomorphic AlInN/sapphire samples with slightly different In contents (13.5, 16.7, and $19.2 \%$ ). The obtained PL peak energies are in good agreement with the data of Wang et al [30]. In order to determine the bowing parameter in the whole $x$ range, additional PL data for $x=0$ and for $x>0.7$ were taken from the work of Kamimura et al [56]. A similar expression as in (13) was applied for bowing parameter $\left(b_{2}(x)\right)$ evaluation. In Eq. 13, we note index $\mathrm{j}=\mathrm{PL}$ and the end point at $x=0$ is expressed as $E_{\mathrm{PL}}^{\mathrm{AlN}}=E_{\mathrm{A}}^{\mathrm{AlN}}+\Delta_{\mathrm{cf}}^{\mathrm{AlN}}$, and at $x=1$ as $E_{\mathrm{PL}}^{\mathrm{InN}}=E_{\mathrm{A}}^{\mathrm{InN}}$. Fitting the experimental data with this expression, we obtain 
Table 5. Band gap bowing parameters for transition energy $E_{\mathrm{A}}$ and PL peak energy $E_{\mathrm{PL}}$.

\begin{tabular}{lcc}
\hline & bowing for $E_{\mathrm{A}}$ & bowing for $E_{\mathrm{PL}}$ \\
\hline$b=\mathrm{const}$ & $5.36 \pm 0.36 \mathrm{eV}$ & $6.49 \pm 0.83 \mathrm{eV}$ \\
$b_{1}(x)=\frac{A}{1+C \cdot x}$ & $\mathrm{~A}=7.77 \pm 0.34 \mathrm{eV}$ & $\mathrm{A}=12.12 \pm 0.95 \mathrm{eV}$ \\
& $\mathrm{C}=1.36 \pm 0.21$ & $\mathrm{C}=2.14 \pm 0.44$ \\
$b_{2}(x)=\frac{A}{1+C \cdot x^{2}}$ & $\mathrm{~A}=6.43 \pm 0.12 \mathrm{eV}$ & $\mathrm{A}=9.31 \pm 0.48 \mathrm{eV}$ \\
& $\mathrm{C}=1.21 \pm 0.14$ & $\mathrm{C}=1.71 \pm 0.34$ \\
& & \\
\hline
\end{tabular}

$\mathrm{A}=9.31 \pm 0.48 \mathrm{eV}$ and $\mathrm{C}=1.71 \pm 0.34$. Experimental data points and fitted bowing curves for transition energy $E_{\mathrm{A}}$ and $\mathrm{PL}$ peak energy $E_{\mathrm{PL}}$ are presented in Fig. 10.

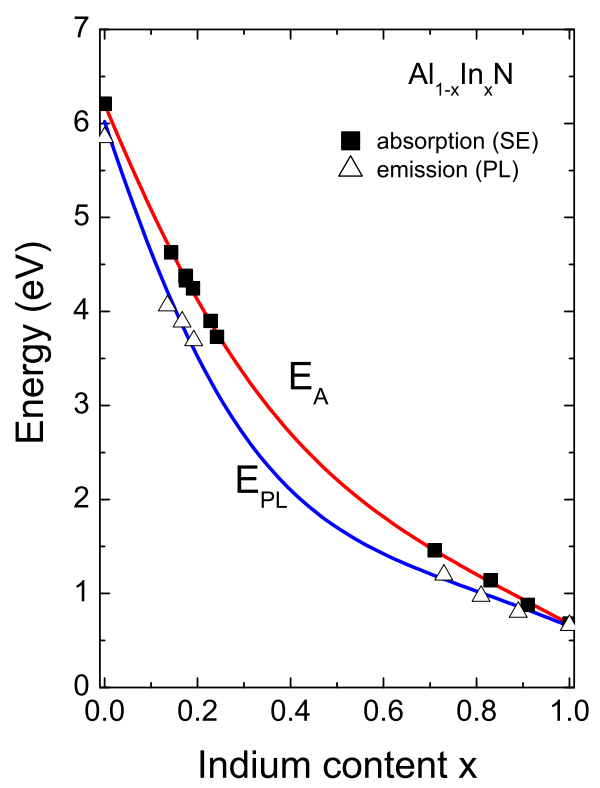

Figure 10. Transition energy $E_{\mathrm{A}}$ and PL peak energy $E_{\mathrm{PL}}$ : experimental and fitted data with bowing parameter $b_{2}(x)$.

The fitted bowing parameter $b_{2}(x)$ values for transition energy $E_{\mathrm{A}}$ and PL peak energy $E_{\mathrm{PL}}$ are summarized in Table 5 together with the conventional bowing parameter $b=$ const and bowing parameter $b_{1}(x)=A /(1+C \cdot x)$ which was proposed by Iliopoulos et al [32]. From the evaluated errors of the fitted parameters, we can deduce that the empirical expression of bowing parameter as a function of In content $b_{2}(x)$ describes the experimental data points with the smallest fitting error.

Our obtained bowing parameter $b_{2}(x)$ ranges from 2.91 to $6.43 \mathrm{eV}$ and yields the value of $4.94 \mathrm{eV}$ for $x=0.5$, which is in good agreement with the $a b$ initio calculated bowing parameter for the uniform AlInN case, which ranges from 2.1 to $6.2 \mathrm{eV}$, and is 
equal to $4.4 \mathrm{eV}$ for $x=0.5$ [57]. Wang et al conducted PLE measurements and obtained a bowing parameter to be about $6 \mathrm{eV}$ (for $0.13<\mathrm{x}<0.24$ ), which is also consistent with our bowing parameter $b_{2}(x)$ for transition energy $E_{\mathrm{A}}$ which yields a value of $6.19 \mathrm{eV}$ for $x=0.18$.

\subsection{Dispersion below the band gap and high-frequency dielectric constant}

In this section we will derive the values of high-frequency dielectric constant $\varepsilon_{\infty}$ and deduce its linear interpolation or AlInN material nearly lattice-matched to GaN.

Following the Kramers-Kronig relation, the peak of $\varepsilon_{1}$ redshifts for the samples with higher In content. The dispersion of $\varepsilon_{1}$ for AlInN samples in the transparent region (below the band gap) can be presented by simplified analytical expression [58]:

$$
\varepsilon_{1}(\hbar \omega)=1+\frac{2}{\pi}\left(\frac{A_{\mathrm{G}}}{2} \ln \frac{E_{\mathrm{H}}^{2}-(\hbar \omega)^{2}}{E_{\mathrm{G}}^{2}-(\hbar \omega)^{2}}+\frac{A_{\mathrm{H}} E_{\mathrm{H}}}{E_{\mathrm{H}}^{2}-(\hbar \omega)^{2}}\right),
$$

where energies $E_{\mathrm{G}}$ and $E_{\mathrm{H}}$ denote average band gap and the high-energy transitions, with their amplitudes $A_{\mathrm{G}}$ and $A_{\mathrm{H}}$, respectively.

The high-frequency dielectric constant $\left(\varepsilon_{\infty}\right)$ is obtained by fitting experimentally obtained $\varepsilon_{1}$ below the band gap with the analytical expression (15) and by extrapolating this expression to zero photon energy $(\hbar \omega \rightarrow 0)$. Their values together with the fitted parameters are represented in Tab. 6. In Fig. 11 the $\varepsilon_{1}$ values are presented as a function of photon energy for AlInN samples together with AlN and GaN for comparison. The precise $\varepsilon_{1}$ (or refractive index values $n$ ) values are needed for optoelectronic device development.

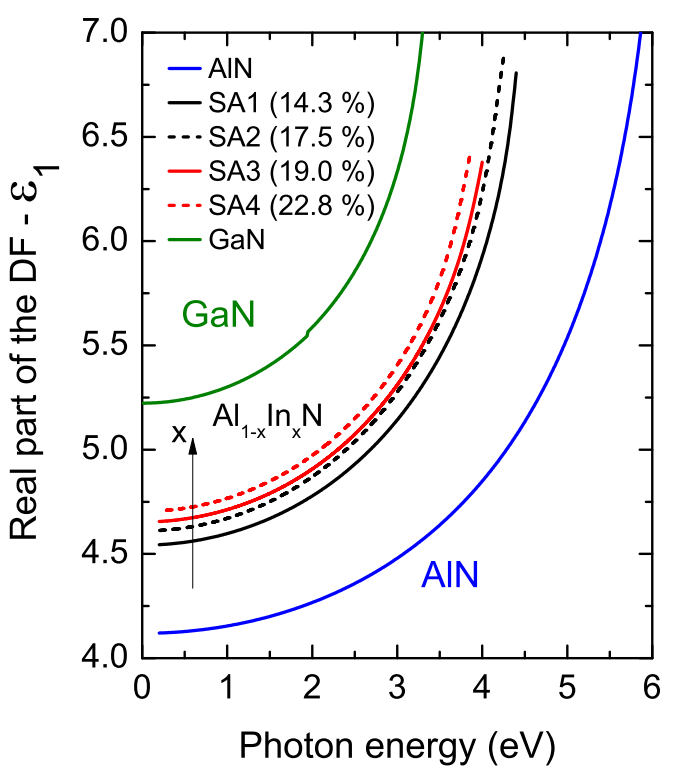

Figure 11. Real part of the DF $\left(\varepsilon_{1}\right)$ in the transparent region (below the band gap). 
Table 6. High-frequency dielectric constant and fitted parameters

\begin{tabular}{lccccc}
\hline Sample & $\varepsilon_{\infty}$ & $A_{\mathrm{G}}$ & $\begin{array}{c}E_{\mathrm{G}} \\
(\mathrm{eV})\end{array}$ & $\begin{array}{c}A_{\mathrm{H}} \\
(\mathrm{eV})\end{array}$ & $\begin{array}{c}E_{\mathrm{H}} \\
(\mathrm{eV})\end{array}$ \\
\hline AlN & 4.11 & 2.91 & 6.16 & 34.90 & 10.26 \\
SA1 $(14.3 \%)$ & 4.54 & 1.53 & 4.52 & 40.01 & 8.81 \\
SA2 $(17.5 \%)$ & 4.61 & 1.82 & 4.38 & 38.85 & 8.82 \\
SA3 $(19.0 \%)$ & 4.65 & 1.47 & 4.21 & 41.15 & 8.87 \\
SA4 $(22.8 \%)$ & 4.71 & 1.40 & 4.02 & 41.63 & 8.82 \\
SI1 $(17.4 \%)$ & 4.61 & 2.47 & 4.37 & 36.71 & 10.43 \\
SI2 $(24.2 \%)$ & 4.67 & 2.34 & 3.85 & 36.73 & 11.31 \\
\hline
\end{tabular}

By extrapolating obtained $\varepsilon_{\infty}$ values for $x<0.2$, the following expression of high frequency dielectric constant as a function of In content for AlInN alloys is obtained:

$$
\varepsilon_{\infty}=4.12 \cdot x+2.78
$$

By deriving the $\varepsilon_{1}$ values from Eq. 15 for $480 \mathrm{~nm}$ wavelength and assuming that $n=\sqrt{\varepsilon_{1}}$ bellow the band gap, we obtain the expression of AlInN index contrast to GaN at room-temperature as a linear function of In content $(x)$, extrapolated for $x<0.2$ :

$$
\Delta n / n=0.29 \cdot x-0.125
$$

where $\Delta n / n=\left(n^{\mathrm{AlInN}}-n^{\mathrm{GaN}}\right) / n^{\mathrm{GaN}}$ and $n^{\mathrm{GaN}}=2.43$ at $480 \mathrm{~nm}$ wavelength. The slope and intercept in Eq. 17 were evaluated with the error of \pm 0.03 and \pm 0.006 , respectively. For $x=0.18(\mathrm{x}=0) \Delta n / n$ yields the value of $-7.28 \%(-12.50 \%)$, which is slightly different in comparison with the value of $-6.40 \%(-12.70 \%)$ obtained by using the expression (2) in Ref. [59].

\section{Summary}

The effective ordinary dielectric function and its analytical model of $\mathrm{Al}_{1-x} \operatorname{In}_{x} \mathrm{~N}$ layers nearly lattice-matched to GaN were determined in the photon energy range from 1 to $10 \mathrm{eV}$ at the room temperature. The excitonic binding energy was estimated to be about $30 \mathrm{meV}$ for AlInN nearly LM to GaN. The absorption onset of the imaginary dielectric function (near the band gap) is red-shifted for the samples with higher In content. Three critical points of the band structure were detected in the high-photon energy range which emphasize already good quality of $\mathrm{Al}_{1-x} \operatorname{In}_{x} \mathrm{~N}$ films nearly lattice-matched to GaN. The strain influence on the band gap was evaluated and the bowing parameter as a function of In content was proposed. Finally, the high-frequency dielectric constants were evaluated. 


\section{Acknowledgments}

For the PL measurements, we would like to thank M. V. Rzheutski, Dr. E. V. Lutsenko, and Prof. G. P. Yablonskii from Stepanov Institute of Physics, National Academy of Sciences of Belarus. The authors acknowledge the financial support of the EC under Grant agreement N:PITN-GA-2008-213238 (RAINBOW project: Initial Training Network of the 7th RTD Framework), Thüringer Kultusministerium (EFRE, B71508015), Bundesministerium für Bildung und Forschung (Grants Nos. 05KS4KTB/3 and 05ES3XBA/5), and Deutsche Forschungsgemeinschaft (SFB 787 framework).

\section{References}

[1] J. Kuzmik. Power electronics on InAlN/(In)GaN: Prospect for a record performance. IEEE Electron Device Lett., 22:510, 2001.

[2] A. Dadgar, M. Neuburger, F. Schulze, J. Bläsing, A. Krtschil, I. Daumiller, M. Kunze, K.-M. Günther, H. Witte, A. Diez, E. Kohn, and A. Krost. High-current AlInN/GaN field effect transistors. Phys. Status Solidi A, 202:832, 2005.

[3] N. Sarazin, E. Morvan, M. A. di Forte Poisson, M. Oualli, C. Gaquiere, O. Jardel, O. Drisse, M. Tordjman, M. Magis, and S. L. Delage. AlInN/AlN/GaN HEMT Technology on SiC With $10-\mathrm{W} / \mathrm{mm}$ and 50\% PAE at $10 \mathrm{GHz}$. IEEE Electron Device Lett., 31:11, 2010.

[4] J. Xie, X. Ni, M. Wu, J. H. Leach, Ü. Özgür, and H. Morkoç. High electron mobility in nearly lattice-matched AlInN/AlN/GaN heterostructure field effect transistors. Appl. Phys. Lett., 91:132116, 2007.

[5] A. Teke, S. Gökden, R. Tülek, J. H. Leach, Q. Fan, J. Xie, Ü. Özgür, H. Morkoç, S. B. Lisesivdin, and E. Özbay. The effect of AlN interlayer thicknesses on scattering processes in lattice-matched AlInN/GaN two-dimensional electron gas heterostructures. New J. of Phys., 11:063031, 2009.

[6] E. Feltin, J.-F. Carlin, J. Dorsaz, G. Christmann, R. Butté, M. Laügt, M. Ilegems, and N. Grandjean. Crack-free highly reflective AlInN/AlGaN Bragg mirrors for UV applications. Appl. Phys. Lett., 88:051108, 2006.

[7] A.-T. Cheng, Y.-K. Su, and W.-C. Lai. Improved Light Output of Nitride-Based Light-Emitting Diodes by Lattice-Matched AlInN Cladding Structure. IEEE Photonics Technol. Lett., 20:970, 2008.

[8] Z. T. Chen, Y. Sakai, J. C. Zhang, T. Egawa, J. J. Wu, H. Miyake, and K. Hiramatsu. Effect of strain on quantum efficiency of InAlN-based solar-blind photodiodes. Appl. Phys. Lett., 95:083504, 2009.

[9] W.Y. Wengs. AlInN resistive ammonia gas sensors. Sens. Actuators, B, 140:139, 2009.

[10] C. Hums, A. Gadanecz, A. Dadgar, J. Bläsing, P. Lorenz, S. Krischok, F. Bertram, A. Franke, J. A. Schäfer, J. Christen, and A. Krost. AlInN/GaN based multi quantum well structures growth and optical properties. Phys. Status Solidi C, 6:S451, 2009.

[11] A. Altoukhov, J. Levrat, E. Feltin, J.-F. Carlin, A. Castiglia, R. Butté, and N. Grandjean. High reflectivity airgap distributed Bragg reflectors realized by wet etching of AlInN sacrificial layers. Appl. Phys. Lett., 95:191102, 2009.

[12] M. Bellanger, V. Bousquet, G. Christmann, J. Baumberg, and M. Kauer. Highly Reflective GaNBased Air-Gap Distributed Bragg Reflectors Fabricated Using AlInN Wet Etching. Appl. Phys. Express, 2:121003, 2009.

[13] C. Xiong, P. R. Edwards, G. Christmann, E. Gu, M. D. Dawson, J. J. Baumberg, R. W. Martin, and I. M. Watson. RAPID COMMUNICATION: High-reflectivity GaN/air vertical distributed Bragg reflectors fabricated by wet etching of sacrificial AlInN layers. Semicond. Sci. Technol., 25:032001, 2010. 
[14] D. Simeonov, E. Feltin, A. Altoukhov, A. Castiglia, J.-F. Carlin, R. Butté, and N. Grandjean. High quality nitride based microdisks obtained via selective wet etching of AlInN sacrificial layers. Appl. Phys. Lett., 92:171102, 2008.

[15] I. M. Watson, C. Liu, E. Gu, M. D. Dawson, P. R. Edwards, and R. W. Martin. Use of AlInN layers in optical monitoring of growth of GaN-based structures on free-standing GaN substrates. Appl. Phys. Lett., 87:151901, 2005.

[16] R. Butté, J.-F. Carlin, E. Feltin, M. Gonschorek, S. Nicolay, G. Christmann, D. Simeonov, A. Castiglia, J. Dorsaz, H. J. Buehlmann, S. Christopoulos, G. Baldassarri Höger von Högersthal, A. J. D. Grundy, M. Mosca, C. Pinquier, M. A. Py, F. Demangeot, J. Frandon, P. G. Lagoudakis, J. J. Baumberg, and N. Grandjean. REVIEW ARTICLE: Current status of AlInN layers latticematched to GaN for photonics and electronics. J. Phys. D: Appl. Phys., 40:6328, 2007.

[17] W. Walukiewicz, J. W. Ager III, K. M. Yu, Z. Lilienthal-Weber, J. Wu, S.X. Li, R.E. Jones, and J.D. Denlinger. Structure and electronic properties of InN and In-rich group III-nitride alloys. J. Phys. D: Appl. Phys., 39:R83, 2006.

[18] P. Schley, R. Goldhahn, G. Gobsch, M. Feneberg, K. Thonke, X. Wang, and A. Yoshikawa. Influence of strain on the band gap energy of wurtzite InN. Phys. Status Solidi B, 246:1177, 2009 .

[19] A. Sedhain, N. Nepal, M. L. Nakarmi, T. M. Al tahtamouni, J. Y. Lin, H. X. Jiang, Z. Gu, and J. H. Edgar. Photoluminescence properties of AlN homoepilayers with different orientations. Appl. Phys. Lett., 93:041905, 2008.

[20] M. Feneberg, R. A. R. Leute, B. Neuschl, K. Thonke, and M. Bickermann. High excitation and high resolution photoluminescence spectra of bulk AlN. Phys. Rev. B, 82:xxxxxx, 2010, in press.

[21] R. Goldhahn, P. Schley, A. T. Winzer, M. Rakel, C. Cobet, N. Esser, H. Lu, and W. J. Schaff. Critical points of the band structure and valence band ordering at the $\Gamma$ point of wurtzite $\operatorname{InN}$. J. Cryst. Growth, 288:273, 2006.

[22] J. Bhattacharyya, S. Ghosh, M. R. Gokhale, B. M. Arora, H. Lu, and W. J. Schaff. Polarized photoluminescence and absorption in A-plane InN films. Appl. Phys. Lett., 89:151910, 2006.

[23] P. Schley, J. Räthel, E. Sakalauskas, G. Gobsch, M. Wieneke, J. Bläsing, A. Krost, G. Koblmüller, J. S. Speck, and R. Goldhahn. Optical anisotropy of A- and M-plane InN grown on free-standing GaN substrates. Phys. Status Solidi A, 207:1062, 2010.

[24] L. Chen, B. J. Skromme, R. F. Dalmau, R. Schlesser, Z. Sitar, C. Chen, W. Sun, J. Yang, M. A. Khan, M. L. Nakarmi, J. Y. Lin, and H.-X. Jiang. Band-edge exciton states in AlN single crystals and epitaxial layers. Appl. Phys. Lett., 85:4334, 2004.

[25] G. Rossbach, M. Röppischer, P. Schley, G. Gobsch, C. Werner, C. Cobet, N. Esser, A. Dadgar, M. Wieneke, A. Krost, and R. Goldhahn. Valence-band splitting and optical anisotropy of AlN. Phys Satus Solidi B, 247:1679, 2010.

[26] F. Fuchs, C. Rödl, A. Schleife, F. Bechstedt. Efficient $O\left(N^{2}\right)$ approach to solve the Bethe-Salpeter equation for excitonic bound states. Phys. Rev. B, 78:085103, 2008.

[27] R. Goldhahn, P. Schley, A. T. Winzer, G. Gobsch, V. Cimalla, O. Ambacher, M. Rakel, C. Cobet, N. Esser, H. Lu, and W. J. Schaff. Detailed analysis of the dielectric function for wurtzite $\mathrm{InN}$ and In-rich InAlN alloys. Phys. Status Solidi A, 203:42, 2006.

[28] W. Terashima, S. Che, Y. Ishitani, and A. Yoshikawa. Growth and Characterization of AlInN Ternary Alloys in Whole Composition Range and Fabrication of InN/AlInN Multiple Quantum Wells by RF Molecular Beam Epitaxy. Jpn. J. Appl. Phys., 45:539, 2006.

[29] R. E. Jones, R. Broesler, K. M. Yu, J. W. Ager, E. E. Haller, W. Walukiewicz, X. Chen, and W. J. Schaff. Band gap bowing parameter of $\operatorname{In}_{1-x} \mathrm{Al}_{x}$ N. J. Appl. Phys., 104:123501, 2008.

[30] K. Wang, R. W. Martin, D. Amabile, P. R. Edwards, S. Hernandez, E. Nogales, K. P. O'Donnell, K. Lorenz, E. Alves, V. Matias, A. Vantomme, D. Wolverson, and I. M. Watson. Optical energies of AlInN epilayers. J. Appl. Phys., 103:073510, 2008.

[31] T. S. Oh, J. Ock Kim, H. Jeong, Y. S. Lee, S. Nagarajan, K. Y. Lim, C.-H. Hong, and E.-K. Suh. Growth and properties of Al-rich $\operatorname{In}_{x} \mathrm{Al}_{1-x} \mathrm{~N}$ ternary alloy grown on GaN template by 
metalorganic chemical vapour deposition. J. Phys. D: Appl. Phys., 41:095402, 2008.

[32] E. Iliopoulos, A. Adikimenakis, C. Giesen, M. Heuken, and A. Georgakilas. Energy bandgap bowing of InAlN alloys studied by spectroscopic ellipsometry. Appl. Phys. Lett., 92:191907, 2008.

[33] R. Goldhahn, C Buchheim, P. Schley, A. T. Winzer, and H. Wenzel. Optical Constants of Bulk Nitrides. In J. Piprek, editor, Nitride Semiconductor Devices: Principles and Simulation, page 95. Wiley-VCH, Weinheim, 2007.

[34] K. Takeuchi, S. Adachi, K. Ohtsuka. Optical properties of $\mathrm{Al}_{x} \mathrm{Ga}_{1-x} \mathrm{~N}$ alloy. J. Appl. Phys., 107:023306, 2010.

[35] P.Y. Yu and M. Cardona. Fundamentals of semiconductors. Springer-Verlag, Berlin, 1999.

[36] C. Buchheim, R. Goldhahn, M. Rakel, C. Cobet, N. Esser, U. Rossow, D. Fuhrmann, A. Hangleiter. Dielectric function and critical points of the band structure for AlGaN alloys. Phys. Status Solidi B, 242:2610, 2005.

[37] P. Schley, R. Goldhahn, A. T. Winzer, G. Gobsch, V. Cimalla, O. Ambacher, H. Lu, W. J. Schaff, M. Kurouchi, Y. Nanishi, M. Rakel, C. Cobet, N. Esser. Dielectric function and Van Hove singularities for In-rich $\operatorname{In}_{x} \mathrm{Ga}_{1-x} \mathrm{~N}$ alloys: Comparison of N- and metal-face materials. Phys. Rev. B, 75:205204, 2007.

[38] C. Hums, A. Gadanecz, A. Dadgar, J. Bläsing, H. Witte, T. Hempel, A. Dietz, P. Lorenz, S. Krischok, J. A. Schäfer, J. Christen, and A. Krost. MOVPE growth and characterization of AllnN FET structures on $\mathrm{Si}(111)$. In Advances in GaN, GaAs, SiC and related alloys on silicon substrates, volume 1068. Materials Research Society symposium proceedings, 2008.

[39] C. Cobet, R. Goldhahn, W. Richter, N. Esser. Identification of van Hove singularities in the GaN dielectric function: a comparison of the cubic and hexagonal phase. Phys. Status Solidi B, 246:1440, 2009.

[40] V. Darakchieva, M. Beckers, M.-Y. Xie, L. Hultman, B. Monemar, J.-F. Carlin, E. Feltin, M. Gonschorek, and N. Grandjean. Effects of strain and composition on the lattice parameters and applicability of Vegards rule in Al-rich $\mathrm{Al}_{1-x} \mathrm{In}_{x} \mathrm{~N}$ films grown on sapphire. J. Appl. Phys., 103:103513, 2008.

[41] V. Darakchieva, M.-Y. Xie, F. Tasndi, L. Monemar B. Abrikosov, I. A. Hultman, J. Kamimura, and K. Kishino. Lattice parameters, daviations from Vegard's rule, and $E_{2}$ phonons in InAlN. Appl. Phys. Lett., 93:261908, 2008.

[42] W. Paszkowicz, R. Černý, and S. Krukowski. Rietveld refinement for indium nitride in the 105295 k range. Powder Diffr., 18:114, 2003.

[43] V. Darakchieva, B. Monemar, and A. Usui. On the lattice parameters of GaN. Appl. Phys. Lett., 91:031911, 2007.

[44] W. Paszkowicz, S. Podsiadlo, and R. Minikayev. Rietveld-refinement study of aluminium and gallium nitrides. J. Alloys Compd., 382:100, 2004.

[45] A.F. Wright. Elastic properties of zinc-blende and wurtzite AlN, GaN, and InN. J. Appl. Phys., $82: 2833,1997$.

[46] S. Shokhovets, L. Spieß, and G. Gobsch. Spectroscopic ellipsometry of wurtzite ZnO and GaN: Examination of a special case. J. Appl. Phys., 107:023509, 2010.

[47] R. Goldhahn. Dielectric function of nitride semiconductors: recent experimental results. Acta Phys. Pol. A, 104:123, 2003.

[48] R. Goldhahn and S. Shokhovets. Optoelectronic Properties of Semiconductors and Superlattices. In M.O. Manasreh and H.X. Jiang, editors, III -Nitride Semiconductors: Optical Properties II, volume 14, page 73. Taylor \& Francis Books, New York, 2002.

[49] J.J. Hopfield. Fine structure in the optical absorption edge of anisotropic crystals. J. Phys. Chem. Solids, 15:97, January 1960.

[50] P. Carrier and S.-H. Wei. Theoretical study of the band-gap anomaly of InN. J. Appl. Phys., 97:033707, 2005.

[51] D. E. Aspnes. Direct Verification of the Third-Derivative Nature of Electroreflectance Spectra. 
Phys. Rev. Lett., 28:168, 1972.

[52] S. L. Chuang and C. S. Chang. $k \cdot p$ method for strained wurtzite semiconductors. Phys. Rev. B, 54:2491, 1996.

[53] Q. Yan, P. Rinke, M. Scheffler, and C. G. van de Walle. Strain effects in group-III nitrides: Deformation potentilas for AlN, GaN, and InN. Appl. Phys. Lett., 95:121111, 2009.

[54] J. Bhattacharyya, S. Ghosh, and H. T. Grahn. Optical polarization properties of interband transitions in strained group-III-nitride alloy films on GaN substrates with nonpolar orientation. Appl. Phys. Lett., 93:051913, 2008.

[55] I. Vurgaftman and J. R. Meyer. Band parameters for nitrogen-containing semiconductors. J. Appl. Phys., 94:3675, 2003.

[56] J. Kamimura, T. Kouno, S. Ishizawa, A. Kikuchi, and K. Kishino. Growth of high-In-content InAlN nanocolums on $\mathrm{Si}(111)$ by RF-plasma-assisted molecular-beam epitaxy. J. Cryst. Growth, 300:160, 2007.

[57] I. Gorczyca, S. P. Łepkowski, T. Suski, N. E. Christensen, and A. Svane. Influence of indium clustering on the band structure of semiconducting ternary and quaternary nitride alloys. Phys. Rev. B, 80:075202, 2009.

[58] S. Shokhovets, R. Goldhahn, G. Gobsch, S. Piekh, R. Lantier, A. Rizzi, V. Lebedev, and W. Richter. Determination of the anisotropic dielectric function for wurtzite AlN and GaN by spectroscopic ellipsometry. J. Appl. Phys., 94:307, 2003.

[59] J.-F. Carlin, C. Zellweger, J. Dorsaz, S. Nicolay, G. Christmann, E. Feltin, R. Butté, and N. Grandjean. Progresses in III-nitride distributed Bragg reflectors and microcavities using AlInN/GaN materials. Phys. Status Solidi B, 242:2326, 2005. 